\title{
BMJ Open Emotion regulation group therapy for deliberate self-harm: a multi-site evaluation in routine care using an uncontrolled open trial design
}

\author{
Hanna Sahlin, ${ }^{1}$ Johan Bjureberg, ${ }_{1}^{1}$ Kim L Gratz, ${ }^{2}$ Matthew T Tull, ${ }^{2}$ Erik Hedman, ${ }^{1}$ \\ Jonas Bjärehed, ${ }^{3}$ Jussi Jokinen, ${ }^{1,4}$ Lars-Gunnar Lundh, ${ }^{3}$ Brjánn Ljótsson, ${ }^{1}$ \\ Clara Hellner ${ }^{1}$
}

To cite: Sahlin H, Bjureberg J, Gratz KL, et al. Emotion regulation group therapy for deliberate self-harm: a multisite evaluation in routine care using an uncontrolled open trial design. BMJ Open 2017;7:e016220. doi:10.1136/ bmjopen-2017-016220

- Prepublication history for this paper is available online. To view these files please visit the journal online (http://dx.doi org/10.1136/bmjopen-2017016220).

Received 1 February 2017 Revised 16 June 2017 Accepted 10 July 2017

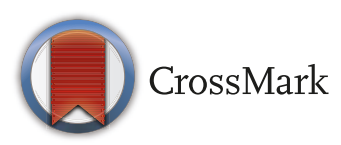

${ }^{1}$ Department of Clinical Neuroscience, Karolinska Institutet, Stockholm, Sweden ${ }^{2}$ Department of Psychology, University of Toledo, Toledo, Ohio, USA

${ }^{3}$ Department of Psychology, Lund University, Lund, Sweden

${ }^{4}$ Department of Clinical

Sciences, Psychiatry, Umeå University, Umeå, Sweden

Correspondence to

Hanna Sahlin;

Hanna.Sahlin@ki.se

\section{ABSTRACT}

Objective Emotion regulation group therapy (ERGT) has shown promising results in several efficacy trials. However, it has not been evaluated outside a research setting. In order to increase the availability of empirically supported treatments for individuals with borderline personality disorder and deliberate self-harm, an evaluation of ERGT in routine clinical care was conducted with therapists of different professional backgrounds who had received brief intensive training in ERGT prior to trial onset.

Design Multi-site evaluation, using an uncontrolled open trial design with assessments at pretreatment, posttreatment and 6-month follow-up.

Setting 14 adult outpatient psychiatric clinics across Sweden.

Participants Ninety-five women (mean age $=25.1$ years) with borderline personality disorder (both threshold and subthreshold) and repeated self-harm were enrolled in the study. Ninety-three per cent of participants completed the post-treatment assessment and $88 \%$ completed the follow-up assessment.

Primary and secondary outcome measures Primary outcome was self-harm frequency as measured with the Deliberate Self-Harm Inventory. Secondary outcomes included self-harm versatility, emotion dysregulation, other self-destructive behaviours, depression, anxiety, stress symptoms and interpersonal and vocational difficulties. Intervention ERGT is an adjunctive, 14-week, acceptance-based behavioural group treatment that directly targets both self-harm and its proposed underlying mechanism of emotion dysregulation.

Results At post-treatment, intent-to-treat analyses revealed a significant improvement associated with a moderate effect size on the primary outcome of self-harm frequency $(51 \%$, reduction; Cohen's $d=0.52, p<0.001)$ as well as significant improvements in the secondary outcomes of self-harm versatility, emotion dysregulation, other self-destructive behaviours and general psychiatric symptomatology. These results were either maintained or further improved on at 6-month follow-up.

Conclusions ERGT appears to be a feasible, transportable and useful treatment for deliberate self-harm and other self-destructive behaviours, emotion dysregulation and psychiatric symptoms when delivered by clinicians in the community.
Strengths and limitations of this study

- This multi-site evaluation in routine clinical care suggests that emotion regulation group therapy may be an easily disseminated and useful treatment for deliberate self-harm.

- Participants reported continued reductions in deliberate self-harm after treatment conclusion.

- This study lacked a control group, limiting our ability to draw conclusions about the effectiveness of emotion regulation group therapy specifically.

- As only adult women were included in the study, generalisability to other patient populations is unclear.

Trial registration number NCT01986257; results.

\section{INTRODUCTION}

Deliberate self-harm (DSH; also referred to as non-suicidal self-injury) is defined as 'the deliberate, direct destruction or alteration of body tissue without conscious suicidal intent, but resulting in injury severe enough for tissue damage (eg, scarring) to occur'. ${ }^{1}$ DSH is highly prevalent in both clinical and non-clinical adult and adolescent populations ${ }^{2-4}$ and is particularly common among individuals with borderline personality disorder $\left(\mathrm{BPD}^{56}\right)$. DSH has been implicated in the high levels of healthcare utilisation among individuals with $\mathrm{BPD}^{7}$ and is one of the strongest predictors of future suicide attempts. ${ }^{89}$

Although the past 2 decades have seen the development of several efficacious treatments for DSH within BPD, including dialectical behaviour therapy $\left(\mathrm{DBT}^{6}\right)$, mentalisation-based treatment ${ }^{10}$ and emotion regulation group therapy $\left(\mathrm{ERGT}^{11}{ }^{12}\right.$ ), few studies have evaluated these treatments in traditional clinical settings (for exceptions, see Bales $e t$ 
$a l$, Feigenbaum et al, Pasieczny et al, Wagner $e t a l^{13-16}$ ), and the extent to which they can be disseminated to community clinicians remains unclear. ${ }^{17}$ Indeed, although efficacy trials are imperative for establishing the evidence base of a treatment, they often maximise internal validity, specialised training and experimental controls at the cost of external validity and applicability to traditional clinical settings. ${ }^{18} 19$ Trials of treatments provided in routine clinical settings, on the other hand, can evaluate how a treatment works under more real-world conditions and, as such, contribute important information about the utility and feasibility of a treatment in traditional clinical settings. These trials also play an important role in increasing the availability of evidence-based treatments. ${ }^{20}$ Thus, further research examining the utility of empirically supported treatments for DSH within BPD in realworld clinical settings is needed.

This study sought to examine the utility of one such treatment in a nationwide open trial. Specifically, in an effort to increase the availability of clinically feasible treatments for DSH in BPD within the community, we conducted an evaluation of ERGT (a 14-week adjunctive group treatment with established efficacy in the treatment of DSH within $\mathrm{BPD}^{12}$ ) in routine clinical care, as delivered by community clinicians at 14 psychiatric outpatient clinics throughout Sweden. Consistent with past research on ERGT, we expected to find significant improvements from pretreatment to post-treatment in DSH and other self-destructive behaviours, emotion dysregulation, psychiatric symptoms and adaptive functioning, as well as stability of these improvements during the 6-month follow-up period.

\section{MATERIALS AND METHODS \\ Design and participants}

The present trial was conducted at 14 psychiatric outpatient clinics located throughout Sweden. We used an uncontrolled open trial design with a 6-month follow-up.

Participants were recruited and assessed by community-based healthcare professionals at the psychiatric outpatient clinics. To ensure comparability of the findings to previous ERGT trials, the inclusion criteria in this study were similar to those used in earlier ERGT studies. ${ }^{11} 1220$ Eligibility criteria included: (a) being a woman $\geq 18$ years, (b) meeting $\geq 3$ diagnostic criteria for BPD as determined by the Structured Clinical Interview for DSM-IV Personality Disorders $\left(\mathrm{SCID}_{\mathrm{II}}{ }^{21}\right)$, (c) $\geq 3$ episodes of DSH in the past 6 months as assessed by a clinician-administered interview version of the Deliberate Self-Harm Inventory $\left(\mathrm{DSHI}^{1}\right),(\mathrm{d})$ ongoing treatment as usual in the community and (e) stability of psychotropic medications for at least 2 months before inclusion. Exclusion criteria were minimal and included only: (a) a DSM-IV ${ }^{22}$ diagnosis of psychotic or bipolar I disorder or ongoing (past month) substance dependence as assessed with the MINI International Neuropsychiatric Interview (MINI 6 ${ }^{23}$ ), (b) the presence of co-occurring psychiatric disorders that required immediate treatment (eg, anorexia nervosa), (c) insufficient understanding of the Swedish language and (d) current life circumstances that would interfere with treatment (ie, being homeless). The study was approved by the Regional Ethical Review Board in Stockholm (Dnr 2013/1321-31/3) and was registered on Clinicaltrials.gov (Identifier NCT01986257). Due to an administrative error, the study was not released on the Clinical Trials platform by the principal investigator, until November 4, 4weeks after the first patient was included. At that date, one group had had one ERGT session and another group had had two ERGT sessions, but there were no changes in the study protocol at any time between the start of inclusion of patients and the release of the protocol.

Between October 2013 and March 2014, 108 female participants with threshold or subthreshold BPD were considered for participation in the study. All participants provided written informed consent. Eight participants did not meet inclusion criteria, four dropped out before completing the pretreatment assessment and one completed the pretreatment assessment but died from suicide before beginning ERGT. Thus, the final sample size was 95 participants. Diagnostic and demographic data for the final sample are presented in table 1. Notably, this sample was comparable to those of both past ERGT trials and other BPD treatment outcome studies with regard to both demographic characteristics and co-occurring psychiatric disorders. ${ }^{12} 15202425$ Participant flow through the trial is described in figure 1.

\section{Selection of participating clinics and study therapists}

An invitation to participate in the study was distributed through a national network of psychiatric caregivers (with representatives from all county councils). Thirty-two clinics responded to the invitation, from which 15 clinics were selected. All participating clinics had to have at least one employed therapist meeting the following criteria: (a) educated within a regulated profession (eg, a licensed nurse, psychologist or psychotherapist) and (b) basic training in cognitive behavioural therapy (with training in acceptance and commitment therapy (ACT) and/or DBT preferred). Consideration was also given to the clinics' geographical location, aiming for as broad a national geographical representation as possible. One included clinic did not participate in the study due to local administrative difficulties, leaving 14 clinics and 28 therapists in the study. Across these clinics, a total of 17 groups were conducted. Median number of participants treated at each clinic was seven (IQR: 5-9; $\min 4$, $\max 11$ ). The clinics were located in 9 cities (population size range: 33 155-2 136 042, median $\left.101615.5^{26}\right)$.

To compensate for the extra cost of implementing a new treatment within the context of regular care, the clinics received monetary compensation for administration of ERGT at a value of US $\$ 1800$ per group. 
Table 1 Sociodemographic, clinical and diagnostic data of the sample $(n=95)$

\begin{tabular}{llllll}
\hline Variable & $\mathbf{n}$ & $\mathbf{( \% )}$ & Mean & SD & Range \\
\hline Age & & & 25.1 & 7.0 & $18-49$
\end{tabular}

Educational level

$\begin{array}{lll}\text { Primary school } & 27 & (28.4) \\ \text { High school/vocational school } & 58 & (61.1) \\ \text { University } & 10 & (10.5)\end{array}$

Marital status

$\begin{array}{lll}\text { Single } & 26 & (27.3) \\ \text { Married/cohabiting } & 24 & (25.3) \\ \text { Living with children } & 16 & (16.8) \\ \text { Occupational status } & & \\ \text { Full-time student } & 37 & (39.0) \\ \text { Employed } & 24 & (25.3) \\ \text { Unemployed } & 21 & (22.1) \\ \text { On disability pension } & 13 & (13.7) \\ \text { On temporary sick leave } & 24 & (25.3)\end{array}$

Clinical characteristics

Meeting full diagnostic criteria for BPD

Number of threshold BPD criteria

$65 \quad(68.0)$

Suicide attempt, lifetime

Suicide attempt, past 3 months*

$54 \quad(58.1)$

DSH frequency past 6 months

$18 \quad(17.2)$

Rate of psychiatric medication use

68

(71.6)

Number of psychiatric medications

80

Rate of previous psychiatric treatment

80

Months of ongoing treatment

Type of ongoing treatment

Cognitive behavioural therapy

30

Psychodynamic therapy

Supportive therapy

Other

45

Co-occurring psychiatric disorders

$\begin{array}{lcl}\text { Depression } & 51 & (53.7) \\ \text { Panic disorder } & 36 & (37.9) \\ \text { Social anxiety disorder } & 36 & (37.9) \\ \text { Post-traumatic stress disorder } & 20 & (21.1) \\ \text { Generalised anxiety disorder } & 38 & (40) \\ \text { Eating disorder } & 15 & (15.8) \\ \text { Substance use disorders } & 4 & (4.2)\end{array}$

BPD, borderline personality disorder; DSH, deliberate self-harm.

${ }^{\star}$ There were data missing for two participants on history of attempted suicide $(n=93)$.

\section{Assessments}

Clinician-administered assessments at baseline included the BPD module of SCID-II, ${ }^{21}$ an interview version of DSHI, ${ }^{1}$ MINI $6^{23}$ and the Columbia-Suicide Severity Rating Scale. ${ }^{27}$ Treatment outcome measures were administered in self-report format at baseline, pretreatment, post-treatment and 6-month follow-up. All self-report measures used in the study were completed online (a method with demonstrated validity ${ }^{28}$ ).

The primary outcome measure was the total frequency of DSH measured by DSHI, a self-report measure with adequate test-retest reliability and construct, discriminant 


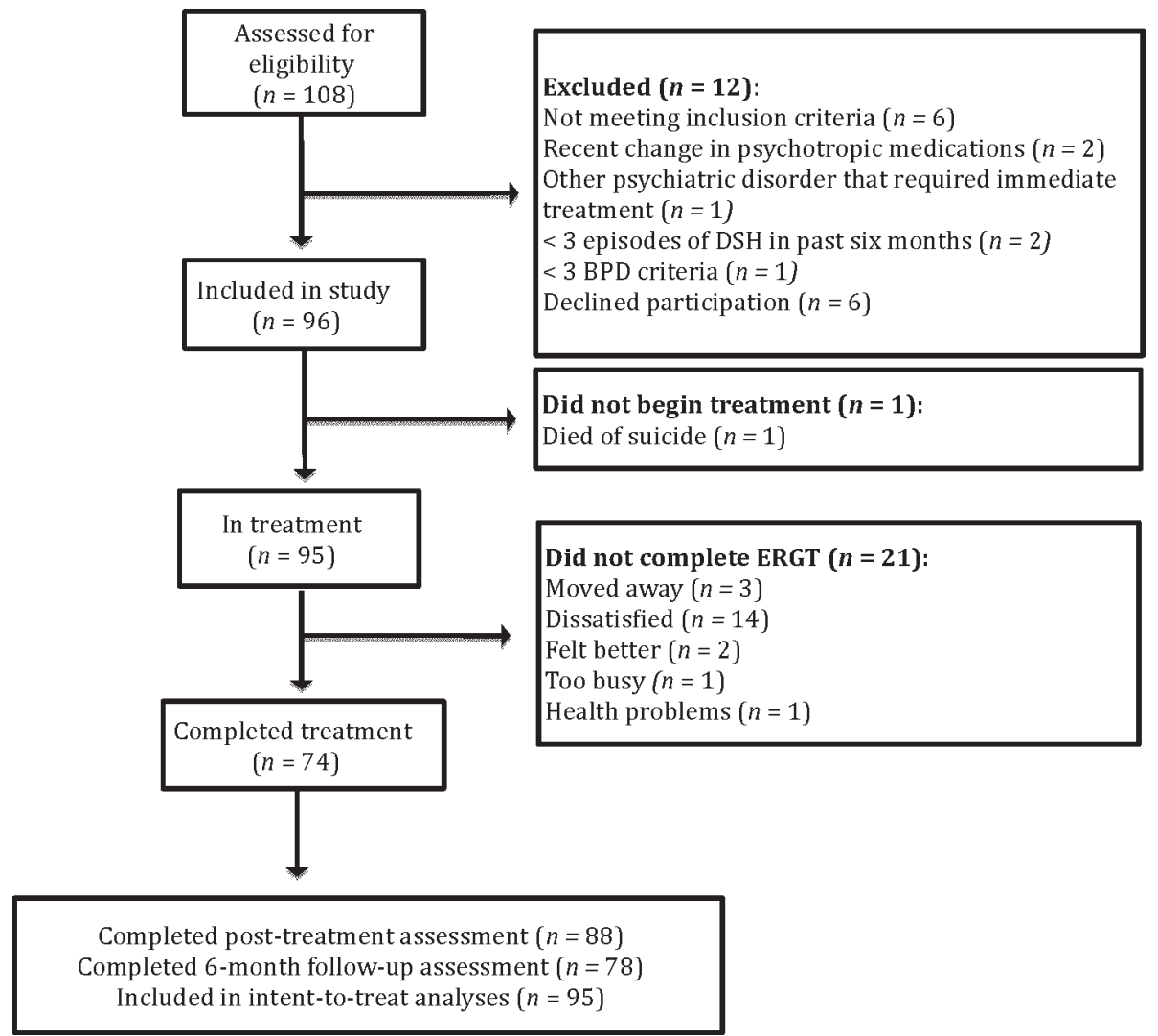

Figure 1 Participant flow through the study. BPD, borderline personality disorder; DSH, deliberate self-harm; ERGT, emotion regulation group therapy.

and convergent validity. ${ }^{1}$ DSHI specifies 16 different types of DSH (eg, cutting, burning or hitting oneself). DSHI was also used to assess DSH versatility (ie, number of different types of DSH behaviours in the past 4 months)—an index of DSH severity. ${ }^{29}$

The secondary outcome measures included the following self-report measures: the Difficulties in Emotion Regulation Scale $\left(\right.$ DERS $^{30}$ ), a measure of clinically relevant emotion regulation difficulties $(\alpha=0.90$ in this sample) with good test-retest reliability and construct and predictive validity ${ }^{20} 3031$; the 11-item self-destructive behaviour supplement to the Borderline Symptom List $\left(\mathrm{BSL}^{32}\right)$, which assesses past week engagement in several self-destructive behaviours (eg, binge eating, excessive drinking, drug use, risky sexual behaviours); the 21-item Depression Anxiety Stress Scales, ${ }^{33}$ a measure of depression, anxiety and stress symptoms $(\alpha=0.88$ in this sample) with good test-retest reliability and construct and discriminant validity ${ }^{33}$; the BPD-related composite of the Inventory of Interpersonal Problems ${ }^{35}$ a measure of BPD-relevant interpersonal difficulties $(\alpha=0.89$ in this sample) with good convergent validity and specificity ${ }^{35}$; and the Sheehan Disability Scales, ${ }^{36}$ a widely used measure of social and vocational impairment due to psychological symptoms ( $\alpha=0.80$ in this sample) with adequate reliability and construct, convergent and discriminant validity across various clinical populations. ${ }^{37}{ }^{38}$ Treatment credibility and expectancy were assessed after the second session of ERGT with the Credibility/Expectancy Questionnaire. ${ }^{39}$

\section{Treatment}

ERGT is a 14-session, adjunctive, acceptance-based behavioural group treatment developed to treat DSH by targeting its underlying mechanism of emotion dysregulation. ${ }^{11}$ Based on Gratz and Roemer's ${ }^{30}$ conceptualisation of emotion regulation, ERGT systematically teaches skills aimed at improving a number of dimensions of emotion regulation, including: emotional awareness, understanding and acceptance; the ability to control behaviours when experiencing negative emotions; the use of non-avoidant emotion regulation strategies to modulate the intensity and/or duration of emotional responses; and the willingness to experience negative emotions as part of pursuing meaningful activities in life. Moreover, the following themes are emphasised throughout the treatment: (a) the potentially paradoxical effects of emotional avoidance; (b) the emotion regulating consequences of emotional acceptance and willingness; and (c) the importance of controlling behaviour when emotions are present, rather than controlling emotions themselves. A detailed description of the content and development of ERGT is available elsewhere. ${ }^{11}$

The ERGT treatment manual (Gratz and Tull, 2010, unpublished manual) provides thorough instructions on the therapeutic stance and theoretical framework of 
ERGT, as well as detailed descriptions of the content, in-session exercises and homework assignments for each session. Sudden deterioration or suicidal crises are monitored weekly through self-report measures assessing DSH frequency and emotion dysregulation and addressed by the group therapists in collaboration with the ongoing treatment provider if needed. The ERGT treatment manual was translated into Swedish through a collaborative and iterative process involving the treatment developer (KLG) and co-author of the ERGT manual (MTT) and the primary ERGT supervisors for this trial (HS and JB, in collaboration with LGL). In this trial, ERGT was delivered in weekly 2-hour sessions led by two therapists with groups of 4-9 participants (average group size $=5.59)$.

\section{Therapist training and treatment fidelity}

Prior to the trial, therapists were required to study the ERGT manual and relevant articles on the theoretical underpinnings of ERGT, as well as to participate in a 3-day workshop led by the authors of the ERGT manual (KLG and MTT). The workshop consisted of didactic lectures, demonstrations, role playing and practice exercises. Of the 28 therapists, 22 were licensed psychologists, 2 were social workers, 2 were nurses, 1 was a psychiatric aid and 1 was an occupational therapist. Twenty-six therapists had previous experience treating patients with BPD and/or DSH; 3 therapists had previous experience with ACT, and 19 therapists had experience with DBT. To ensure treatment fidelity during the ERGT trial, all sessions were filmed and reviewed weekly by clinicians with experience delivering ERGT (HS, JB), and all therapists received the option of weekly supervision based on the reviewed films.

\section{Statistics and data analysis}

We expected to recruit 90-105 participants. This sample size yielded high power $(>0.99$ with $\alpha=0.05)$ to detect a standardised mean difference between pretreatment and post-treatment of $\mathrm{d}=0.5$ using within-group Student's t-tests on a log-transformation of the primary outcome measure DSH frequency (consistent with past research on $\mathrm{ERGT}^{111220}$ ).

All analyses were performed in $\mathrm{R}$ using random effects modelling. ${ }^{40}$ The count variables, DSH frequency and BSL, are reported with medians and IQR and were analysed using negative binomial generalised mixed models, and the remaining continuous outcomes are reported as means and SD and were analysed using linear models. The models included all available data at the three assessment points (pretreatment, post-treatment and 6-month follow-up) for each outcome, thus making them intentto-treat analyses. We estimated separate slopes for the change between the pretreatment and post-treatment assessments ( $\mathrm{S} 1)$ and the change between the post-treatment and 6-month follow-up assessments (S2). Random intercepts and random slopes as well as autoregressive correlation structures were included in the models if they significantly improved model fit according to log-likelihood ratio tests.

Effect sizes were calculated for changes between pretreatment, post-treatment and 6-month follow-up. For the count variables (ie, DSHI frequency and BSL), the percentage change from baseline to any subsequent time point with $95 \% \mathrm{CI}$ was used as an effect size. This was calculated by exponentiating the estimate for the slopes derived from the negative binomial models and interpreting the range below or above 1 as the percentage decrease or increase in the outcome for a 1-unit increase in the predictor. Effect sizes for the remaining continuous outcomes are reported as Cohen's d, calculated by dividing the appropriate slope estimate (ie, pretreatment to post-treatment: S1; pretreatment to 6-month follow-up: $\mathrm{S} 1+\mathrm{S} 2$; and post-treatment to 6-month follow-up: S2) by the pretreatment SD. We also performed separate analyses where linear mixed models were applied to log-transformed DSH frequency and BSL scores and corresponding effect sizes were extracted. These effect sizes are reported together with observed means and SD of DSH frequency and BSL scores to permit comparison with previous studies of ERGT. ${ }^{11} 12{ }^{20}$ However, inferences of the statistical significance of changes on these measures were based on the more appropriate negative binomial regression models. CIs with a $95 \%$ margin for the effect sizes were calculated using 5000 bootstrap replications. ${ }^{41}$ The bootstrap replications were clustered on participants. ${ }^{42}$

We also performed sensitivity analyses to investigate the robustness and validity of the DSHI results. First, the treating clinics were entered as random factors to test the possibility of clustering effects in the data. Second, we added concurrent medication status (coded as 0 for no concurrent medication and 1 for concurrent medication) and type of treatment as usual (coded as a factor with the following levels: cognitive behavioural therapy, psychodynamic therapy, supportive therapy or other) as covariates in the model. These covariates were added both as simple effects and as interaction effects with the S1 variable to investigate the possible influence of the covariates on DSH frequency and treatment effect. Third, we entered the number of treatment sessions attended as a predictor of improvement in DSH frequency during the treatment period.

Finally, we examined the number of participants who reported no (zero) DSH episodes at each assessment point and used McNemar's exact tests to analyse the changes between the assessment points.

\section{RESULTS}

\section{Treatment adherence and attrition}

The average time from baseline assessment to the start of treatment was 20.7 days $(\mathrm{SD}=17.2$, range $1-91)$. Twenty-one participants (22\%) dropped out of ERGT (see figure 1). Mean number of sessions attended for all included participants was $11(\mathrm{SD}=5.2, \min 0, \max 16)$. 
Seventy-two participants $(76 \%)$ attended $\geq 7$ sessions, and $47(49 \%)$ attended 14 sessions. Post-treatment assessments were completed by $88(93 \%)$ participants, and 6-month follow-up assessments were completed by $76(82 \%)$ participants. Mean ratings of treatment credibility and expectancy completed after the second session were $5.7(\mathrm{SD}=1.8)$ and $47.0 \%(\mathrm{SD}=25.5)$, respectively (for comparison, mean ratings reported by Gratz and Tull were $6.91 \%$ and $57 \%$, respectively). ${ }^{20}$

\section{Primary outcome}

Results for both the primary and secondary outcome measures are displayed in table 2. There was a significant $52 \%$ reduction in DSH frequency from pretreatment to post-treatment and a $76 \%$ reduction from pretreatment to 6-month follow-up.

The observed means for DSH frequency were 53.68 $(\mathrm{SD}=99.88), 37.45(\mathrm{SD}=72.22)$ and $28.69(\mathrm{SD}=95.44)$ at pretreatment, post-treatment and follow-up, respectively. Effect sizes (Cohen's d) based on log-transformed data showed medium-sized reductions in DSH frequency from pretreatment to post-treatment $(\mathrm{d}=0.52,95 \% \mathrm{CI} 0.30$ to 0.75$)$ and from post-treatment to 6-month follow-up $(\mathrm{d}=0.47,95 \%$ CI 0.27 to 0.70$)$. A large effect size was observed from pretreatment to 6-month follow-up $(\mathrm{d}=0.99,95 \%$ CI 0.70 to 1.30$)$.

The percentage of participants with an observed score of zero DSH episodes during the past 4 months increased significantly from $4.2 \%$ at pretreatment to $17.9 \%$ at post-treatment (Exact McNemar's $\chi^{2}=12.25$, $\mathrm{df}=1, \mathrm{p}<0.001$ ) and to $25.3 \%$ at follow-up (Exact McNemar's $\left.\chi^{2}=16.67, \mathrm{df}=1, \mathrm{p}<0.001\right)$. The increase between post-treatment and follow-up was also significant (Exact McNemar's $\chi^{2}=7.14, \mathrm{df}=1, \mathrm{p}=0.01$ ).

\section{Secondary outcomes}

There were significant improvements in DSH versatility, emotion dysregulation, self-destructive behaviours and depression and stress symptoms at post-treatment, accompanied by small to large effect sizes (see table 2). At the 6-month follow-up, all of these improvements were either maintained or further improved on, with the change on DERS from pretreatment to 6-month follow-up reaching a large-sized effect. Median reduction in self-destructive behaviours over each time period is reported in table 2 . The observed mean self-destructive behaviour scores were $4.82(\mathrm{SD}=3.69), 3.65(\mathrm{SD}=4.24)$ and $3.24(\mathrm{SD}=3.61)$ at pretreatment, post-treatment and follow-up, respectively. Analyses on log-transformed data showed small to medium effect sizes between pretreatment and post-treatment (Cohen's $\mathrm{d}=0.43,95 \%$ CI 0.20 to 0.65 ) and pretreatment and 6-month follow-up ( $\mathrm{d}=0.55,95 \%$ CI 0.27 to $0.77)$. Improvements in interpersonal difficulties, anxiety and social and vocational impairment were not significant at post-treatment; however, at 6-month follow-up, there were significant improvements in these outcomes (accompanied by small to moderate effect sizes) from pretreatment (stemming from the additional significant improvements in these measures observed from post-treatment to 6-month follow-up).

\section{Sensitivity analyses}

First, we included the treating clinics as random factors in the DSH frequency model. This did not improve model fit, suggesting that there was not a significant clustering effect of treating clinic in the data. Second, we included concurrent medication status and type of treatment as usual as simple effects and interaction effects with S1 (ie, the change between the pretreatment and post-treatment assessments) in the DSH frequency model. None of the added predictors were statistically significant $(p>0.05)$, suggesting that concurrent medication use and type of treatment as usual were not associated with treatment effect. Third, we included the number of attended sessions as a predictor of improvement in DSH frequency. The interaction effect between session count and S1 was statistically significant, $(\mathrm{B}=0.06, \mathrm{Z}=11.54, \mathrm{p}<0.001)$, indicating that higher attendance was associated with larger improvements during treatment.

\section{DISCUSSION}

The present multi-site evaluation of ERGT in routine clinical care provides additional support for the feasibility and transportability of this treatment. Results revealed significant improvements in DSH frequency and versatility, emotion dysregulation, self-destructive behaviours and depression and stress symptoms from pretreatment to post-treatment. By the 6-month follow-up, interpersonal difficulties and social and vocational impairment had also improved significantly. Moreover, all gains found at post-treatment were either maintained or further improved on at follow-up. Notably, the results of the sensitivity analyses strengthen our confidence in these findings, providing evidence of a significant effect of session attendance, but not treatment clinic, concurrent medication use or type of treatment as usual, on improvements in DSH frequency. These results are consistent with past findings that characteristics of participants' ongoing therapy in the community had minimal impact on treatment response to $\mathrm{ERGT}^{43}$ and suggest that it is engagement in ERGT rather than other (non-specific) treatment-related factors that influences reductions in DSH frequency. Nonetheless, the uncontrolled open trial design precludes conclusions regarding the causal relation of treatment participation to symptom improvement as there may be other factors underlying both treatment completion status and symptom improvement (ie, motivation for treatment or alliance with treatment providers) that may, at least in part, account for the present findings.

Although the results of this study need to be interpreted with caution (due to the lack of a control group), they are similar to those obtained in previous ERGT efficacy trials. ${ }^{11}{ }^{12}$ Likewise, our findings of either stability or further improvements during the follow-up period are consistent with the observed pattern of continued 


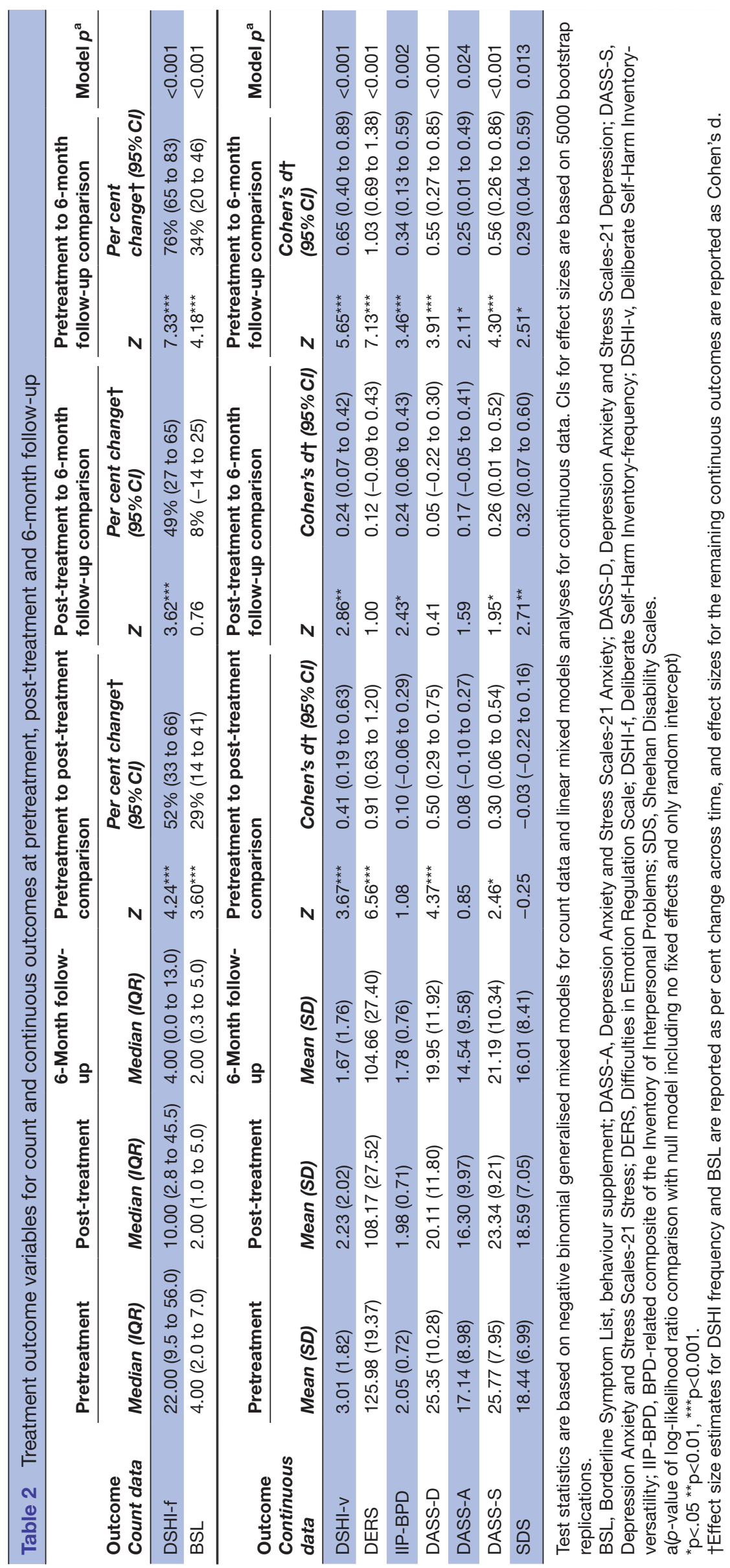


or maintained improvement during a 9-month follow-up period in Gratz and colleagues' randomised controlled trial (RCT) ${ }^{12}$ These findings provide further support for the durability of improvements following this relatively brief and non-intensive treatment, suggesting that participants may experience durable gains through ERGT even when delivered by community clinicians with only brief training in this treatment.

Despite these similarities with previous ERGT trials, the pattern of findings for measures of interpersonal, social and vocational functioning differed from Gratz and colleagues' previous ERGT studies. ${ }^{12}{ }^{20}$ Specifically, results revealed no significant changes in interpersonal difficulties or social and vocational impairment from pretreatment to post-treatment, although there were significant (although small) improvements in these areas during the follow-up period. Although the differences in these findings may be due to study-related differences in therapist training and/or treatment delivery, they may also be explained (in part) by the fact that more than one-third of participants (39\%) in the present trial were on disability pension or current sick leave, thus limiting their social interactions and vocational opportunities during treatment.

Notably, rates of abstinence from DSH increased significantly from pretreatment to post-treatment, as well as from post-treatment through the 6-month follow-up, with $25.2 \%$ of participants reporting abstinence from DSH 6 months post-treatment. Nonetheless, it warrants mention that the proportion of participants reaching abstinence from DSH was lower than in previous ERGT trials. ${ }^{120}$ This may be due to differences in the level of training and supervision provided in this trial versus previous trials. Indeed, ratings of treatment credibility and expectancy in this trial were somewhat lower than in previous studies of ERGT, potentially capturing the lesser experience of the therapists in this trial. Conversely, the lower rate of treatment-related abstinence from DSH observed in this study may reflect differences in sample composition and/or clinical severity (as both emotion dysregulation and DSH frequency reported in this sample were at the high end of the range reported in past ERGT studies). Nonetheless, past research examining predictors of treatment response to ERGT found that several indicators of greater severity in domains relevant to ERGT (ie, baseline emotion dysregulation and BPD criteria, lifetime and recent DSH, and past-year hospitalisation and suicide attempts) predicted better responses during treatment and follow-up, ${ }^{43}$ suggesting that greater severity in certain domains may be associated with better response to this treatment. Future research is needed to clarify the particular patients most likely to benefit from ERGT.

Importantly, we found significant reductions across all assessment points in DSH versatility (ie, a marker of DSH severity associated with future suicide risk). ${ }^{29}$ This finding lends support to the potential utility of ERGT in decreasing risk for self-injurious behaviours in general. Indeed, given the high occurrence of DSH and suicidal behaviours, ${ }^{9}$ as well as evidence that DSH is one of the strongest prospective predictors of future suicide attempts, ${ }^{44} 45$ the emphasis within ERGT on reducing DSH could be expected to reduce suicidal risk as well. Nonetheless, in the absence of data in this or previous ERGT trials on suicidal outcomes in particular, the benefits of ERGT for suicidality remain unknown and in need of future investigation.

Although results of this study provide preliminary support for the utility of ERGT in routine clinical care, they also highlight more broadly the potential utility of targeting emotion dysregulation in the treatment of BPD-related pathology. Notably, there are several other empirically supported treatments for BPD and related pathology that directly target emotion dysregulation, including $\mathrm{DBT}^{6}$ and Systems Training for Emotional Predictability and Problem Solving. ${ }^{46}$ Findings of the efficacy of those treatments for BPD, ${ }^{24} 4647$ combined with both the results of the current study and past findings supporting the efficacy of ERGT for DSH within BPD, ${ }^{11} 12$ highlight the potential benefits of interventions aimed at promoting adaptive emotion regulation among individuals with BPD and provide further support for emotion dysregulation as a primary mechanism underlying the pathogenesis and effective treatment of BPD and related pathology. ${ }^{48}$

There are several strengths of this study that are worth noting. First, the naturalistic design of this study permitted inclusion of a large sample of participants who received this ERGT as part of their standard treatment. Second, data attrition was low ( $8 \%$ at post-treatment and $18 \%$ at follow-up) and participant dropout was within expected levels when treating individuals with $\mathrm{BPD}^{49}$ and consistent with previous ERGT trials. ${ }^{12}$ Third, contrary to previous ERGT trials, ${ }^{11} 1220$ the group leaders were of different professional backgrounds and representative of the community clinicians who regularly treat this patient population, providing support for the generalisability and transportability of this treatment. Finally, our data provide support for the feasibility of disseminating ERGT to community clinicians, as therapists in this study were provided with only readings and a brief workshop on ERGT prior to its delivery.

Despite these strengths, findings must be considered in light of limitations present. First, the lack of a control condition precludes conclusions about the effects of this ERGT (vs treatment as usual or the passage of time). However, it is important to note that the waitlist conditions in previous ERGT trials generally evidenced stability over time on all measures of interest (likely due to the relatively short time-frame of the treatment period). Nonetheless, future studies are needed to evaluate the effectiveness of ERGT within an RCT design. Second, even though the participating clinicians were offered weekly supervision based on filmed sessions, no systematic adherence ratings were conducted. This limits our ability to speak to the quality of ERGT and its relation to outcome. Third, the results relied solely on self-report 
measures and not clinician-rated outcomes. However, research on self-reported versus clinician-rated outcomes in psychotherapy studies suggests that the use of self-report measures often results in smaller effect sizes when compared with clinician-administered instruments. ${ }^{50}$ Thus, it is possible that our results may be conservative estimates of improvements during and after treatment. Fourth, our study only included women, which limits the generalisability of the results to men. Future research is needed that evaluates ERGT within male or mixedgender samples. Finally, current (past month) substance dependence was an exclusion criterion in this study, both to ensure comparability with previous ERGT trials and because active substance dependence requires specialised treatment and a higher level of care. Indeed, in Sweden, individuals with ongoing substance dependence are not necessarily offered treatment within a psychiatric clinic; rather, they may be referred to specialised substance dependence treatment centres. Thus, it is unlikely that the exclusion of such individuals negatively affected the generalisability of this sample to a typical Swedish psychiatric clinic. Furthermore, this criterion did not exclude individuals with past (or even recent) substance use problems. Nonetheless, given the high co-occurrence of substance dependence and $\mathrm{BPD},{ }^{51}$ excluding these individuals may have limited the external validity of the sample to patients with BPD as a whole.

Despite these limitations, our results provide further evidence for the utility and transportability of ERGT, suggesting that this is a feasible treatment for DSH, emotion dysregulation and psychiatric symptoms when delivered by community clinicians in traditional clinical settings.

Acknowledgements The authors wish to thank Ida Janson, Sara Eivergård, Niclas Andersson and all study therapists for their hard work and enthusiasm, which made this study possible.

Contributors HS, JB, BL and CH had full access to all of the data in the study and take responsibility for the integrity of the data and the accuracy of the data analysis. Study concept and design: HS, JB, KLG, MT, EH, JOB, LGL, JJ, BL and $\mathrm{CH}$. HS, JB, JOB, BL and CH. Drafting of the manuscript: $\mathrm{HS}, \mathrm{JB}, \mathrm{BL}$ and $\mathrm{CH}$. Critical revision of the manuscript for important intellectual content and approval of the final version to be published: HS, JB, KLG, MT, EH, JOB, LGL, JJ, BL and $\mathrm{CH}$.

Funding This research was supported by the National Self Harm project in Sweden and Stockholm County Council regional research grant \#SLL20140428. None of the funding organisations has had any role in the design and conduct of the study; in the collection, management and analysis of the data; or in the preparation, review and approval of the manuscript.

Competing interests HS, JBju, KLG, MTT, JBjä, L-GL, JJ and CH report no competing interests. EH and BL are shareholders of a company, Dahlia, specialised in psychiatric symptom assessment.

Patient consent All data are anonymised and only presented on group level.

Ethics approval Regional Ethical Review Board in Stockholm.

Provenance and peer review Not commissioned; externally peer reviewed.

Data sharing statement No additional data are available.

Open Access This is an Open Access article distributed in accordance with the Creative Commons Attribution Non Commercial (CC BY-NC 4.0) license, which permits others to distribute, remix, adapt, build upon this work non-commercially, and license their derivative works on different terms, provided the original work is properly cited and the use is non-commercial. See: http://creativecommons.org/ licenses/by-nc/4.0/

(C) Article author(s) (or their employer(s) unless otherwise stated in the text of the article) 2017. All rights reserved. No commercial use is permitted unless otherwise expressly granted.

\section{REFERENCES}

1. Gratz KL. Measurement of Deliberate Self-Harm: preliminary data on the Deliberate Self-Harm Inventory. J Psychopathol Behav Assess 2001;23:253-63.

2. Briere J, Gil E. Self-mutilation in clinical and general population samples: prevalence, correlates, and functions. Am J Orthopsychiatry 1998;68:609-20.

3. de Klerk S, van Noorden MS, van Giezen AE, et al. Prevalence and correlates of lifetime deliberate self-harm and suicidal ideation in naturalistic outpatients: the Leiden Routine Outcome monitoring study. J Affect Disord 2011;133:257-64.

4. Swannell SV, Martin GE, Page A, et al. Prevalence of nonsuicidal selfinjury in nonclinical samples: systematic review, meta-analysis and meta-regression. Suicide Life Threat Behav 2014;44:273-303.

5. Sansone RA, Wiederman MW, Sansone LA. The Self-Harm Inventory (SHI): development of a scale for identifying self-destructive behaviors and borderline personality disorder. J Clin Psychol 1998;54:973-83.

6. Linehan M. Cognitive-behavioral treatment of borderline personality disorder. New York: Guilford Press, 1993.

7. Zanarini MC. Psychotherapy of borderline personality disorder. Acta Psychiatr Scand 2009;120:373-7.

8. Victor SE, Klonsky ED. Correlates of suicide attempts among selfinjurers: a meta-analysis. Clin Psychol Rev 2014;34:282-97.

9. Hamza CA, Stewart SL, Willoughby T. Examining the link between nonsuicidal self-injury and suicidal behavior: a review of the literature and an integrated model. Clin Psychol Rev 2012;32:482-95.

10. Bateman A, Fonagy P. Effectiveness of partial hospitalization in the treatment of borderline personality disorder: a randomized controlled trial. Am J Psychiatry 1999;156:1563-9.

11. Gratz KL, Gunderson JG. Preliminary data on an acceptance-based emotion regulation group intervention for deliberate self-harm among women with borderline personality disorder. Behav Ther 2006;37:25-35.

12. Gratz KL, Tull MT, Levy R. Randomized controlled trial and uncontrolled 9-month follow-up of an adjunctive emotion regulation group therapy for deliberate self-harm among women with borderline personality disorder. Psychol Med 2014;44:2099-112.

13. Bales D, van Beek N, Smits M, et al. Treatment outcome of 18-month, day hospital mentalization-based treatment (MBT) in patients with severe borderline personality disorder in the Netherlands. J Pers Disord 2012;26:568-82.

14. Feigenbaum JD, Fonagy P, Pilling S, et al. A real-world study of the effectiveness of DBT in the UK National Health Service. Br J Clin Psychol 2012;51:121-41.

15. Pasieczny N, Connor J. The effectiveness of dialectical behaviour therapy in routine public mental health settings: an Australian controlled trial. Behav Res Ther 2011;49:4-10.

16. Wagner T, Fydrich T, Stiglmayr C, et al. Societal cost-of-illness in patients with borderline personality disorder one year before, during and after dialectical behavior therapy in routine outpatient care. Behav Res Ther 2014;61:12-22.

17. Swales MA, Taylor B, Hibbs RA. Implementing dialectical behaviour therapy: programme survival in routine healthcare settings. J Ment Health 2012;21:548-55.

18. Weisz JR, Weiss B, Donenberg GR. The lab versus the clinic. Effects of child and adolescent psychotherapy. Am Psychol 1992;47:1578-85.

19. Wells KB. Treatment research at the crossroads: the scientific interface of clinical trials and effectiveness research. Am J Psychiatry 1999;156:5-10.

20. Gratz KL, Tull MT. Extending research on the utility of an adjunctive emotion regulation group therapy for deliberate self-harm among women with borderline personality pathology. Personal Disord 2011;2:316-26.

21. First MB, Gibbon M, Spitzer RL, et al; Structured Clinical Interview for DSM-IV Axis II Personality Disorders, (SCID-II). Washington, D.C: American Psychiatric Press, Inc, 1997.

22. American Psychiatric Association. Diagnostic and statistical manual of mental disorders. 4th ed. Washington, DC: 2000. Author Text Revision. 
23. Sheehan DV, Lecrubier Y, Sheehan KH, et al. The Mini-International Neuropsychiatric Interview (M.I.N.I.): the development and validation of a structured diagnostic psychiatric interview for DSM-IV and ICD10. J Clin Psychiatry 1998;59:22-33. quiz34-57.

24. Linehan MM, Comtois KA, Murray AM, et al. Two-year randomized controlled trial and follow-up of dialectical behavior therapy vs therapy by experts for suicidal behaviors and borderline personality disorder. Arch Gen Psychiatry 2006;63:757-66.

25. McMain SF, Links PS, Gnam WH, et al. A randomized trial of dialectical behavior therapy versus general psychiatric management for borderline personality disorder. Am J Psychiatry 2009;166:1365-74

26. Statistics Sweden. Population in the country, counties and municipalities on 31/12/2013 and population change in 2013. 2014; http://www.scb.se/en /Yearly-statistics-Municipalities-Counties-andthe-whole-country/370301/

27. Posner K, Brown GK, Stanley B, et al. The Columbia-Suicide Severity Rating Scale: initial validity and internal consistency findings from three multisite studies with adolescents and adults. Am J Psychiatry 2011;168:1266-77.

28. Hedman E, Ljótsson B, Andersson E, et al. Effectiveness and cost offset analysis of group CBT for hypochondriasis delivered in a psychiatric setting: an open trial. Cogn Behav Ther 2010;39:239-50.

29. Turner BJ, Layden BK, Butler SM, et al. How often, or how many ways: clarifying the relationship between non-suicidal self-injury and suicidality. Arch Suicide Res 2013;17:397-415.

30. Gratz KL, Roemer L. Multidimensional assessment of emotion regulation and dysregulation: development, factor structure, and initial validation of the Difficulties in Emotion Regulation Scale. $J$ Psychopathol Behav Assess 2004;26:41-54.

31. Bjureberg J, Liótsson B, Tull MT, et al. Development and validation of a brief version of the Difficulties in Emotion Regulation Scale: the DERS-16. J Psychopathol Behav Assess 2016.38.

32. Bohus M, Limberger MF, Frank U, et al. [Development of the Borderline Symptom List]. Psychother Psychosom Med Psychol 2001;51:201-11.

33. Lovibond PF, Lovibond SH. The structure of negative emotional states: comparison of the Depression Anxiety Stress Scales (DASS) with the Beck Depression and Anxiety Inventories. Behav Res Ther 1995;33:335-43.

34. Antony MM, Bieling PJ, Cox BJ, et al. Psychometric properties of the 42-item and 21-item versions of the depression anxiety stress scales in clinical groups and a community sample. Psychol Assess 1998;10:176-81.

35. Lejuez CW, Daughters SB, Nowak JA, et al. Examining the inventory of interpersonal problems as a tool for conducting analogue studies of mechanisms underlying Borderline Personality Disorder. J Behav Ther Exp Psychiatry 2003;34:313-24.

36. Sheehan DV. The Sheehan Disability Scales. The anxiety disease and how to overcome it. New York City: Charles Scribner and Sons, 1983.
37. Hambrick JP, Turk CL, Heimberg RG, et al. Psychometric properties of disability measures among patients with social anxiety disorder. $J$ Anxiety Disord 2004;18:825-39.

38. Diefenbach GJ, Abramowitz JS, Norberg MM, et al. Changes in quality of life following cognitive-behavioral therapy for obsessivecompulsive disorder. Behav Res Ther 2007;45:3060-8.

39. Devilly GJ, Borkovec TD. Psychometric properties of the credibility/expectancy questionnaire. J Behav Ther Exp Psychiatry 2000;31:73-86.

40. Core Team R. R: a language and environment for statistical computing. Vienna, Austria 2015 http://www. R-project.org/.

41. Kelley K. The effects of nonnormal distributions on confidence intervals around the standardized mean difference: bootstrap and parametric confidence intervals. Educ Psychol Meas 2005;65:51-69.

42. Ren S, Lai H, Tong $\mathrm{W}$, et al. Nonparametric bootstrapping for hierarchical data. J Appl Stat 2010;37:1487-98.

43. Gratz KL, Dixon-Gordon KL, Tull MT. Predictors of treatment response to an adjunctive emotion regulation group therapy for deliberate self-harm among women with borderline personality disorder. Personal Disord 2014;5:97-107.

44. Asarnow JR, Porta G, Spirito A, et al. Suicide attempts and nonsuicidal self-injury in the treatment of resistant depression in adolescents: findings from the TORDIA study. J Am Acad Child Adolesc Psychiatry 2011;50:772-81.

45. Wilkinson P, Kelvin R, Roberts $\mathrm{C}$, et al. Clinical and psychosocial predictors of suicide attempts and nonsuicidal self-injury in the Adolescent Depression Antidepressants and Psychotherapy Trial (ADAPT). Am J Psychiatry 2011;168:495-501.

46. Blum N, St John D, Pfohl B et al. Systems Training for Emotional Predictability and Problem Solving (STEPPS) for outpatients with borderline personality disorder: a randomized controlled trial and 1-year follow-up. Am J Psychiatry 2008;165:468-78.

47. Linehan MM, Korslund KE, Harned MS, et al. Dialectical behavior therapy for high suicide risk in individuals with borderline personality disorder. JAMA Psychiatry 2015;72:475-8.

48. Gratz KL, Weiss NH, Tull MT. Examining emotion regulation as an outcome, mechanism, or target of psychological treatments. Curr Opin Psychol 2015;3:85-90.

49. Barnicot K, Katsakou C, Marougka S, et al. Treatment completion in psychotherapy for borderline personality disorder: a systematic review and meta-analysis. Acta Psychiatr Scand 2011;123:327-38.

50. Cuijpers P, Li J, Hofmann SG, et al. Self-reported versus clinician-rated symptoms of depression as outcome measures in psychotherapy research on depression: a meta-analysis. Clin Psychol Rev 2010;30:768-78.

51. Trull TJ, Sher KJ, Minks-Brown C, et al. Borderline personality disorder and substance use disorders: a review and integration. Clin Psychol Rev 2000;20:235-53. 
Correction: Emotion regulation group therapy for deliberate self-harm: a multi-site evaluation in routine care using an uncontrolled open trial design

Sahlin H, Bjureberg J, Gratz KL, et al. Emotion regulation group therapy for deliberate self-harm: a multi-site evaluation in routine care using an uncontrolled open trial design. BMJ Open 2017;7:e016220. doi: 10.1136/bmjopen-2017-016220.

Authors Hanna Sahlin and Johan Bjureberg contributed equally to the manuscript.

Open Access This is an Open Access article distributed in accordance with the Creative Commons Attribution Non Commercial (CC BY-NC 4.0) license, which permits others to distribute, remix, adapt, build upon this work non-commercially, and license their derivative works on different terms, provided the original work is properly cited and the use is non-commercial. See: http://creativecommons.org/licenses/by-nc/4.0/

C Article author(s) (or their employer(s) unless otherwise stated in the text of the article) 2017. All rights reserved. No commercial use is permitted unless otherwise expressly granted.

BMJ Open 2017;7:e016220corr1. doi:10.1136/bmjopen-2017-016220corr1

CrossMark 